\title{
Contabilidade
}

\section{FATORES DETERMINANTES DA DISTRIBUIÇÃO DOS SALÁRIOS POR SETOR DE ATIVIDADE NO BRASIL}

\author{
Nome Lindomar Pegorini Daniel \\ Instituição/Afiliação Universidade do Estado de Mato Grosso - UNEMAT \\ País Brasil \\ Contato principal para correspondência. \\ Nome Márcio Balduíno Saraiva \\ Instituição/Afiliação Faculdade de Viçosa \\ País Brasil
}

\section{RESUMO}

O Brasil está entre os países que pior distribuem a renda. Essa desigualdade também pode ser vista entre os diferentes setores da economia e em outras áreas como no nível de educação da população em geral. Sendo a educação apontada como eficiente mecanismo de transferência e redistribuição de renda, o presente artigo analisou como o rendimento no trabalho e o nível de escolaridade estão distribuídos entre os setores primário, secundário e terciário e se a educação é o principal discriminante dos salários. Através da análise discriminante foi possível confirmar a hipótese de que a educação é o principal fator discriminante dos rendimentos nos diferentes setores da economia. Além disso, constatou-se que outros fatores como raça, gênero, e local de residência, apesar de menos influentes, também são importantes para explicar a discriminação de salários.

Palavras Chave: Distribuição de renda. Educação. Análise discriminante.

\begin{abstract}
Brazil is among the countries that worst distribute income even being considered an upper middle-income country. The inequality can also be seen between the different sectors of the economy and other areas as the education level. Since education is identified as an efficient transfer and income redistribution mechanism, this article examined how job earnings and the level of education are distributed among primary, secondary and tertiary sectors and if the education is the main factor that discriminate wages. Using discriminant analysis we confirm the hypothesis that education is the main factor to discriminate job earnings in the different sectors of the economy. Furthermore, we found that other factors such race, gender, and place of residence, although less influential, also are important to explain wage discrimination.
\end{abstract}

Keywords: Income distribution. Education. Discriminant analysis.

Classificação JEL: D31; I24; J31.

\section{INTRODUÇÃO}




\section{Revista \\ UNEMAT de \\ Contabilidade}

v. 9, n. 17,2020

Com renda nacional bruta de U\$ 9.821 dólares per capita em 2017, o Brasil é considerado um país de renda média alta no contexto internacional. Fato estilizado é que para esse patamar o Brasil apresenta uma das piores distribuições de renda quando comparado a países da mesma faixa de renda ou até mesmo com países com renda inferior. Segundo Todaro e Smith (2012), em 2007, enquanto os $20 \%$ mais pobres detinham cerca de $4,1 \%$ da parcela da renda gerada no país, os $20 \%$ mais ricos detinham cerca de $58,7 \%$.

Os altos índices de concentração de renda podem levar à persistência da pobreza (ADAMS, 2004), e geram externalidades negativas como o aumento da desigualdade social, implicando em baixo índice educacional, aumento do desemprego, entre outros entraves que podem afetar significativamente o crescimento e o desenvolvimento da economia do país.

O Brasil tem alcançado melhorias quanto à redução da pobreza e redistribuição de renda, principalmente nas duas últimas décadas. Segundo Neri e Carvalhaes (2008) o Brasil tem apresentado uma robusta redução na desigualdade de renda desde $2001 \mathrm{e}$, por isso, saiu de terceiro para o décimo lugar na classificação da Organização das Nações Unidas (ONU), para os países que pior distribuem a renda nacional.

Apesar dos avanços apresentados para reduzir a desigualdade no país ainda existe um abismo entre os mais ricos e mais pobres. Segundo Todaro e Smith (2012), em 2005, cerca de 18,3\% da população vivia com menos de 2 dólares por dia, considerado pelo Banco Mundial e pelo Programa das Nações Unidas para o Desenvolvimento (PNUD) como a linha da pobreza. Ainda, em 2007, estima-se que 7,8\% da população brasileira vivia com menos de 1 dólar por dia, considerado como a linha da pobreza extrema.

O programa de transferência de renda Bolsa Família tem sido apontado pelo governo como principal responsável pelos avanços na redução da pobreza, contudo segundo Hanushek et al (2001), na presença de externalidades, o investimento em educação como meio de redistribuição de renda leva a resultados Pareto superiores quando comparados a outros mecanismos de transferência de renda como o subsídio em impostos ou em salários (caso do Bolsa Família).

Desde Becker (1962) que os teóricos do Capital Humano argumentam que o investimento em educação, qualificação e treinamento da mão de obra resultam em retornos futuros em termos de maior produtividade e maiores ganhos de renda para os indivíduos. No Brasil, a desigualdade observada na distribuição da renda também pode ser vista no nível de educação e no mercado de trabalho.

Segundo a Pesquisa Nacional por Amostra de Domicílio (PNAD), em 2011, das pessoas com idade acima de 25 anos, ou seja, que tiveram a chance de terminar o ensino médio, apenas $38,9 \%$ possuíam efetivamente esse nível de ensino ou superior. A situação é pior ao se observar que para a mesma faixa etária cerca de $46,7 \%$ dos indivíduos não possuía ao menos o ensino fundamental de 8 anos de estudo. A média de anos de estudo da população economicamente ativa é de 7,7 anos, estnado abaixo dos 13 anos da América Latina, dos 8,6 do Sul e do Oeste Asiático e dos 9,5 dos países desenvolvidos (HANUSHEK; WOESSMANN, 2009).

Além do gap educacional é possível observar desigualdades no mercado de trabalho afetando principalmente as mulheres e os não brancos que recebem menos apesar de ocuparem as mesmas posições e o mesmo nível de educação que homens e brancos (CARVALHO et al, 2006). Ademais, as diferentes dinâmicas dos mercados de trabalho regionais e setoriais podem também exercer influência sobre a desigualdade e distribuição de renda. 


\section{Revista \\ UNEMAT de \\ Contabilidade}

v. 9, n. 17,2020

Historicamente, fatores geográficos também são importantes para explicar a desigualdade no Brasil, como exemplo a Região Nordeste e a zona rural são as que concentram maior proporção de pobreza no país. Ainda segundo Todaro e Smith (2012), outra preocupação em relação à má distribuição de renda no Brasil é que a pobreza afeta principalmente os não brancos e as mulheres.

Portanto, existem evidências suficientes para acreditar que a estrutura de distribuição de renda no Brasil esteja fortemente relacionada às desigualdades no nível de educação e no mercado de trabalho. Nesse caso, o presente estudo se propõe a analisar as relações entre a distribuição do investimento educacional na população e a distribuição dos ganhos (salários).

Do exposto, o objetivo geral desse trabalho é verificar se há relação entre a distribuição de renda e o nível educacional no Brasil. Especificamente, pretendeu-se: a) dividir a população em grupos de rendimento no trabalho por setor de atividade; b) verificar se a educação é o principal fator discriminante de rendimento entre os grupos, em outras palavras, observar se a educação é um mecanismo importante para redistribuição de renda; e c) identificar a existência de discriminação no mercado de trabalho no Brasil, ou seja, verificar se gênero, raça, e local de residência também discriminam rendimentos.

$\mathrm{O}$ artigo encontra-se organizado em mais 3 seções além desta introdução. A seção 2 apresenta a metodologia aplicada para alcançar os objetivos propostos, elucidando o método estatístico empregado e a seleção e o tratamento de variáveis. O procedimento consiste na estimação de funções discriminantes dos salários para os três setores da economia em nível nacional. A seção 3 apresenta os resultados e a discussão do trabalho, observando as características relevantes para discriminação dos salários e associadas a distribuição de renda. Por fim, a seção 4 apresenta as considerações finais.

\section{METODOLOGIA}

\subsection{Estratégia empírica}

Os dados utilizados são provenientes do banco de microdados da PNAD 2011 do Instituto Brasileiro de Geografia e Estatística (IBGE) e referem-se a indivíduos com idade igual ou superior a 16 anos que auferiram rendimento de qualquer tipo de trabalho nos diferentes setores da economia, totalizando 151.018 observações.

Os indivíduos foram divididos entre os setores primário, secundário e terciário de acordo com o trabalho principal. Para formar grupos de rendimento foi utilizada a definição de classes sociais do IBGE$^{1}$, contudo como o interesse reside no rendimento individual algumas considerações foram feitas.

Em primeiro lugar, o IBGE utiliza a renda média familiar para definir classes de rendimento, já para a análise do investimento em educação, desde Mincer (1974) é amplamente difundido na literatura que a variável de interesse é o salário hora. Portanto, ao invés da renda média familiar utiliza-se nesse estudo o salário hora auferido.

A segunda consideração reside no fato de que o IBGE utiliza o salário mínimo como base para distinguir entre classes de rendimento. Como o salário mínimo referente à PNAD 2011 é de $\mathrm{R}$ \$ 545,00 reais, esse valor foi transformado para salário mínimo por hora com base

\footnotetext{
${ }^{1}$ O IBGE define classes de renda baseado na renda média familiar da seguinte forma: até 2 salários mínimos (classe E), entre 2 e 4 salários mínimos (classe D), entre 4 e 10 salários mínimos (classe C), entre 10 e 20 salários mínimos (classe B) e mais de 20 salários mínimos (classe A).
} 


\section{Revista \\ UNEMAT de \\ Contabilidade}

em 44 horas semanais de trabalho. O resultado da alocação dos indivíduos em grupos de rendimento por setor da economia pode ser visto na Tabela 1.

Tabela 1: Grupos de rendimento segundo setor referente ao trabalho principal - 2011*

\begin{tabular}{lccccccc}
\hline Setor de Atividade & até 2 s.m. & de 2 a 4 s.m. & de 4 a 10 s.m. & de 10 a 20 s. m. & mais de 20 s.m. \\
\hline Setor Primário & 83,1 & 10,0 & 4,7 & 1,2 & 1,0 \\
\hline Setor Secundário & 63,5 & 24,8 & 8,8 & 1,8 & 1,1 \\
\hline Setor Terciário & 57,2 & 23,2 & 13,8 & 3,7 & 1,9 \\
\hline
\end{tabular}

Fonte: Resultados da pesquisa. *s.m. (salários mínimos).

Como se pode observar, a desigualdade na distribuição de renda no Brasil é significante, em média $68 \%$ dos indivíduos auferem salário hora inferior a dois salários mínimos hora. A distribuição de renda é mais igualitária no setor terciário onde a média de anos de estudo (9,6 anos) é maior do que no setor secundário ( 7,9 anos), por sua vez é superior a do setor primário (4,2 anos). Esses são os primeiros indícios de que a escolaridade discrimina os ganhos dos indivíduos e a distribuição de renda.

Uma vez definidos os grupos de rendimento é necessário verificar a influência que a escolaridade, proxy para educação, e outras variáveis possuem na definição dos mesmos. Para identificar se a educação é a variável mais influente para discriminar os grupos de rendimento utilizou-se a técnica multivariada de análise discriminante, descrita na seção seguinte. Essa técnica permite a construção de funções discriminantes entre os grupos identificando os fatores mais importantes para alocar os indivíduos em cada grupo.

Além da escolaridade, a literatura sugere que outras variáveis relacionadas ao mercado de trabalho afetam os salários. Variáveis como experiência, gênero, raça, local de residência (rural ou urbano) e região onde trabalha (Sul, Sudeste, Nordeste, Centro Oeste ou Norte) são comumente utilizadas para explicar rendimentos, sugeridas nos trabalhos de Altonji, Blom e Meghir (2012), Souza, Ponczek e Oliva (2011), Corseuil, Santos e Foguel (2001), Carvalho et al. (2006), Mincer (1974), Oaxaca (1973), Silveira (2011) e Becker (1962).

Escolaridade e experiência estão relacionadas ao estoque de capital humano, treinamento e qualificação. A variável escolaridade é definida como anos de estudo, já a experiência é construída de acordo com Mincer (1974) da seguinte forma:

Experiência $=$ idade - anos de estudo -6

Gênero e raça estão geralmente relacionados às diferenças salariais no mercado de trabalho. A variável gênero é dicotômica e assume valor 1 quando o indivíduo é homem, por sua vez o fator raça é uma variável binária com valor igual a 1 quando o indivíduo se auto declara branco. Segundo Glaeser et al (2004) mesmo não existindo uma forte discriminação aparente, pode haver distinção de rendimentos entre homens e mulheres, e entre raças diferentes mesmo desempenhando as mesmas funções com níveis de escolaridade equivalentes.

Já as variáveis local de residência e região onde trabalha estão relacionadas às diferentes dinâmicas e oportunidades no mercado de trabalho. A variável rural é dicotômica e assume valor 1 quando o indivíduo reside na zona rural, já a variável região é categórica assumindo os seguintes valores: 1 para Sul, 2 para Centro Oeste, 3 para Sudeste, 4 para Norte e 5 para a Região Nordeste. 


\section{Revista \\ UNEMAT de \\ Contabilidade}

Está definida então a estratégia empírica para atender aos objetivos do trabalho, a análise discriminante será conduzida sobre as variáveis escolaridade, experiência, gênero, raça, local de residência e região onde trabalha para discriminar entre os grupos de rendimento construídos. Essa análise possibilitou a identificação das variáveis mais importantes para discriminar os grupos de renda e consequentemente a distribuição da mesma.

\subsection{Análise discriminante multivariada}

Para atender ao objetivo proposto pelo trabalho, identificar quais são os principais determinantes dos diferenciais de salários, faz-se uso da técnica estatística multivariada de análise discriminante. Segundo Mingoti (2005), essa técnica consiste em discriminar populações e, ou, classificar novos elementos amostrais em populações pré-definidas. Para isso, determinam-se os pesos de uma combinação linear de duas (ou mais) variáveis independentes que maximiza as diferenças entre os grupos, definidos a priori. Um dos métodos mais utilizados para determinar os coeficientes (pesos) é o método de Fisher (HAIR et al, 2005).

A determinação da função discriminante de Fisher é constituída de quatro etapas. A priori, definem-se os grupos $\left(\tau_{1}\right.$ e $\left.\tau_{2}\right)$ e o número de elementos $\left(\mathrm{n}_{1}\right.$ e $\left.\mathrm{n}_{2}\right)$ dos grupos a serem discriminados. Além do vetor de $\mathrm{p}$ variáveis dos elementos do grupo, representado por $\mathrm{X}^{1}=$ $\left[\mathrm{x}_{1} \mathrm{x}_{2} \mathrm{x}_{3} \cdots \mathrm{x}_{\mathrm{p}}\right]$. Em seguida, define-se a matriz de variância conjunta das populações $\left(\mathrm{S}_{\mathrm{p}}\right)$, que tem a propriedade de representar matrizes de variância e covariância, homogêneas, das variáveis.

$\mathrm{S}_{\mathrm{p}}=\frac{\left(\mathrm{n}_{1}-1\right) \mathrm{S}_{1}+\left(\mathrm{n}_{2}-1\right) \mathrm{S}_{2}}{\mathrm{n}_{1}+\mathrm{n}_{2}-2}$

A função discriminante de Fisher busca determinar uma ou mais combinações lineares das variáveis $\mathrm{x}_{1} \mathrm{x}_{2} \mathrm{x}_{3} \cdots \mathrm{x}_{\mathrm{p}}$, representadas da seguinte forma:

$Y=a^{\prime} x$

No método de Fisher determinam-se os a's que fornecem a maior diferença entre as médias e a menor variância da combinação linear. Então, os valores de Y obtidos para cada elemento de $\tau_{1}$, são o máximo possível separados dos valores de $\mathrm{Y}$, obtidos em $\tau_{2}$. No caso de $\mathrm{g}$ grupos, determinam-se no máximo $(\mathrm{g}-1)$ funções discriminantes. Em que, as funções discriminantes são ordenadas pelo grau de discriminação dos grupos.

\section{RESULTADOS E DISCUSSÃO}

\subsection{Análise descritiva}

A Tabela 2 apresenta a média e o desvio padrão em 2011 das variáveis utilizadas na análise empírica por setor de atividade.

Tabela 2: Resumo de estatísticas das variáveis utilizadas na análise empírica - 2011

\begin{tabular}{lcccccc}
\hline \multirow{2}{*}{ Variáveis } & \multicolumn{2}{c}{ Primário } & \multicolumn{2}{c}{ Secundário } & \multicolumn{2}{c}{ Terciário } \\
\cline { 2 - 7 } & Média & $\begin{array}{l}\text { Desvio } \\
\text { padrão }\end{array}$ & Média & $\begin{array}{l}\text { Desvio } \\
\text { padrão }\end{array}$ & Média & $\begin{array}{c}\text { Desvio } \\
\text { padrão }\end{array}$ \\
\hline $\begin{array}{l}\text { Grupo } \\
\text { até 2 salários mínimos }\end{array}$ & 0,831 & 0,005 & 0,635 & 0,004 & 0,572 & 0,002 \\
& \multicolumn{2}{c}{38} & & & &
\end{tabular}


de 2 a 4 salários mínimos de 4 a 10 salários mínimos de 10 a 20 salários mínimos mais de 20 salários mínimos

Características individuais

Escolaridade

Experiência

Masculino

Branco

\section{Características geográficas}

Rural

Sul

Centro-Oeste

Sudeste

Norte

Nordeste

Fonte: Resultados da pesquisa.

$\begin{array}{llllll}0,100 & 0,003 & 0,248 & 0,003 & 0,232 & 0,001 \\ 0,047 & 0,002 & 0,088 & 0,002 & 0,138 & 0,001 \\ 0,012 & 0,001 & 0,018 & 0,001 & 0,037 & 0,001 \\ 0,001 & 0,001 & 0,001 & 0,001 & 0,002 & 0,001\end{array}$

$\begin{array}{cccccc}4,192 & 0,693 & 7,775 & 4,118 & 9,606 & 4,101 \\ 32,641 & 16,320 & 23,174 & 14,348 & 21,642 & 13,941 \\ 0,866 & 0,340 & 0,777 & 0,416 & 0,483 & 0,499 \\ 0,363 & 0,481 & 0,444 & 0,496 & 0,478 & 0,499\end{array}$

$\begin{array}{llllll}0,659 & 0,473 & 0,070 & 0,256 & 0,048 & 0,214 \\ 0,156 & 0,008 & 0,197 & 0,004 & 0,150 & 0,002 \\ 0,079 & 0,003 & 0,074 & 0,002 & 0,088 & 0,001 \\ 0,250 & 0,011 & 0,470 & 0,005 & 0,469 & 0,003 \\ 0,122 & 0,005 & 0,062 & 0,001 & 0,069 & 0,001 \\ 0,390 & 0,010 & 0,194 & 0,003 & 0,222 & 0,002\end{array}$

As estatísticas descrevem a desigualdade existente na distribuição de renda no país. Como já observado o setor terciário é o que apresenta distribuição de renda menos desigual e também apresenta maior nível de escolaridade média. Os dados revelam também que os setores primário e secundário são predominantemente masculinos, além disso, o setor primário é o que apresenta menor escolaridade média e menor proporção de não brancos trabalhando.

Quanto as características geográficas, é possível verificar que o setor primário é o único com número expressivo de indivíduos residindo na zona rural. Em relação a distribuição de trabalhadores entre os setores, o Nordeste é a região que mais emprega no setor primário (com menores salários e nível de escolaridade), já o Sudeste é o que mais emprega tanto no setor secundário quanto terciário.

As Tabelas 3 e 4 apresentam a distribuição da população empregada e o salário hora médio por gênero, por setor de atividade e por nível de escolaridade em 2011, respectivamente.

Tabela 3: Distribuição dos indivíduos empregados por gênero, por setor da economia e por nível de escolaridade no Brasil - 2011

\begin{tabular}{lc|c|c|c|c|c}
\hline & \multicolumn{3}{c}{ Homens } & & \\
\cline { 2 - 7 } Nível de escolaridade & Primário & Secundário & Terciário & Primário & Secundário & Terciário \\
& & & & & & \\
\hline Fundamental incompleto & 11,51 & 13,00 & 14,71 & 2,36 & 3,65 & 19,09 \\
\hline Fundamental completo & 1,73 & 6,36 & 10,44 & 0,40 & 2,41 & 13,01 \\
\hline Médio completo & 1,29 & 9,21 & 22,73 & 0,43 & 5,16 & 36,78 \\
\hline Superior completo & 0,19 & 1,30 & 6,88 & 0,05 & 1,00 & 14,64 \\
\hline Pós-graduação & 0,01 & 0,06 & 0,58 & 0,00 & 0,04 & 0,98 \\
\hline
\end{tabular}

Fonte: Resultados da pesquisa. 
Como se observa da Tabela 3, as mulheres estão predominantemente empregadas no setor terciário, enquanto que os homens estão mais bem distribuídos entre os diferentes setores. Além disso, como posto anteriormente, os setores primário e secundário são predominantemente masculinos.

Tabela 4: Salário hora médio auferido por gênero, por setor da economia e por nível de escolaridade no Brasil - 2011

\begin{tabular}{lc|c|c|c|c|c}
\hline & \multicolumn{3}{c}{ Homens } & & & \\
\cline { 2 - 7 } Nível de escolaridade & Primário & Secundário & Terciário & Primário & Secundário & Terciário \\
& & & & & & \\
\hline Fundamental incompleto & 4,99 & 6,92 & 7,25 & 3,84 & 4,68 & 5,13 \\
\hline Fundamental completo & 10,10 & 7,70 & 8,16 & 4,81 & 5,16 & 5,73 \\
\hline Médio completo & 12,30 & 11,10 & 12,12 & 9,14 & 7,40 & 8,12 \\
\hline Superior completo & 25,77 & 30,64 & 31,35 & 15,59 & 20,39 & 19,49 \\
\hline Pós-graduação & 44,14 & 86,24 & 55,11 & - & 43,27 & 41,79 \\
\hline
\end{tabular}

Fonte: Resultados da pesquisa.

Com relação a Tabela 4, três importantes fatos podem ser inferidos. Primeiro, que as mulheres ganham em média menos que os homens para o mesmo nível de escolaridade em todos os setores da economia. Segundo, que o rendimento médio tende a aumentar conforme se eleva o nível de escolaridade no mercado de trabalho como um todo. E, por fim, que a discrepância entre os rendimentos de homens e mulheres não se reduz conforme aumenta o nível de escolaridade.

Essas particularidades evidenciam a existência de fatores que determinam de forma divergente como os indivíduos de gêneros distintos se inserem no mercado de trabalho e que definem, também de modo desigual, seus níveis de rendimento. Não apenas o nível de escolaridade e o gênero que são apontados como fatores relevantes para discriminar o salário pessoal, as Tabelas 5, 6 e 7 apresentam o salário hora médio por raça, por gênero e por local de residência e de trabalho para os setores da economia, respectivamente.

Tabela 5: Salário hora médio auferido por raça, por setor da economia e por nível de escolaridade no Brasil - 2011

\begin{tabular}{lc|c|c|c|c|c}
\hline & \multicolumn{3}{c}{ Brancos } & \multicolumn{3}{c}{ Não Brancos } \\
\cline { 2 - 8 } Nível de escolaridade & Primário & Secundário & Terciário & Primário & Secundário & Terciário \\
& & & & & & \\
\hline Fundamental incompleto & 6,14 & 7,02 & 7,19 & 4,11 & 6,23 & 5,56 \\
\hline Fundamental completo & 10,31 & 7,32 & 7,39 & 8,47 & 7,01 & 6,71 \\
\hline Médio completo & 15,94 & 10,45 & 10,96 & 7,10 & 9,60 & 8,89 \\
\hline Superior completo & 29,11 & 27,95 & 25,06 & 10,81 & 24,18 & 22,45 \\
\hline Pós-graduação & 51,75 & 57,00 & 51,08 & 12,04 & 35,17 & 37,46 \\
\hline
\end{tabular}

Fonte: Resultados da pesquisa.

O quadro mostrado na Tabela 5 indica que os brancos empregados nos diferentes setores ganham mais que os não brancos para todos os níveis de escolaridade. Esse fato está 


\section{Contabilidade}

de acordo com o argumento de Glaeser et al (2004) de que, apesar de não aparente, existe discriminação no mercado de trabalho tanto para homens e mulheres quanto para brancos e não brancos. Esse fato pode ser melhor visto nos dados apresentados na Tabela 6 .

Tabela 6: Salário hora médio auferido e escolaridade por gênero, por raça e por setor da economia no Brasil - 2011

\begin{tabular}{|c|c|c|c|c|}
\hline \multirow{2}{*}{ Características Pessoais } & \multicolumn{2}{|c|}{ Homens } & \multicolumn{2}{|c|}{ Mulheres } \\
\hline & Brancos & Não Brancos & Brancas & Não Brancas \\
\hline \multicolumn{5}{|l|}{ Setor Primário } \\
\hline Escolaridade média & 5,0 & 3,6 & 5,5 & 4,2 \\
\hline Salário/hora médio & 8,98 & 5,03 & 6,68 & 3,76 \\
\hline \multicolumn{5}{|l|}{ Setor Secundário } \\
\hline Escolaridade média & 8,4 & 6,9 & 9,4 & 8,4 \\
\hline Salário/hora médio & 11,37 & 8,38 & 8,23 & 6,42 \\
\hline \multicolumn{5}{|l|}{ Setor Terciário } \\
\hline Escolaridade média & 10,0 & 8,6 & 10,6 & 9,1 \\
\hline Salário/hora médio & 15,35 & 10,58 & 11,38 & 7,44 \\
\hline
\end{tabular}

Fonte: Resultados da pesquisa.

É notável que a população branca aufere renda média superior à população não branca, no entanto, a primeira também possui escolaridade média superior à última. Contudo, quando a população masculina é comparada com a feminina, a primeira obtém rendimento superior à última mesmo possuindo nível de escolaridade inferior.

Tabela 7: Salário hora médio auferido por local de residência, por região e por setor da economia no Brasil - 2011

\begin{tabular}{|c|c|c|c|c|c|}
\hline Setor de Atividade & Sul & Sudeste & Centro Oeste & Norte & Nordeste \\
\hline \multicolumn{6}{|l|}{ Setor Primário } \\
\hline Urbano & 11,70 & 7,85 & 21,19 & 6,23 & 5,52 \\
\hline Rural & 6,62 & 5,76 & 5,88 & 5,77 & 3,46 \\
\hline \multicolumn{6}{|l|}{ Setor Secundário } \\
\hline Urbano & 8,23 & 11,64 & 8,27 & 7,69 & 6,14 \\
\hline Rural & 6,78 & 7,50 & 8,65 & 5,39 & 3,92 \\
\hline \multicolumn{6}{|l|}{ Setor Terciário } \\
\hline Urbano & 10,82 & 12,80 & 12,67 & 9,19 & 9,10 \\
\hline Rural & 7,59 & 6,17 & 7,37 & 5,98 & 5,51 \\
\hline
\end{tabular}

Fonte: Resultados da pesquisa.

Já com a Tabela 7 é possível observar que para o setor primário o maior rendimento é observado na Região Centro Oeste, isso se deve ao emprego de alta tecnologia e demanda de mão de obra com maior nível de qualificação. Para os setores secundário e terciário os maiores rendimentos são observados na Região Sudeste, também devido a esses setores serem mais desenvolvidos nessa região e possibilitarem oportunidades de emprego com maior nível de qualificação.

De modo geral os rendimentos para os residentes na zona rural são inferiores que para a população que reside na zona urbana. Adicionalmente, os menores rendimentos para ambos locais de residência são encontrados na Região Nordeste, como afirmado anteriormente essa região, historicamente, é a que concentra maior proporção da população pobre no Brasil. 
Em resumo, os fatores utilizados na análise parecem discriminar de forma satisfatória os salários nos diferentes setores no Brasil. A próxima seção, portanto, concentra-se na estimação das funções e das cargas discriminantes para determinar qual a característica mais importante para diferenciar os grupos de rendimento.

\subsection{A educação como mecanismo de transferência de renda}

Como colocado anteriormente, a principal hipótese do artigo é que a educação é o fator mais relevante para discriminar os grupos de rendimento no Brasil e, portanto, seria um eficiente mecanismo de transferência de renda como indicado por Hanushek et al (2001). Para testar essa hipótese um estudo de análise discriminante foi conduzido sobre os grupos de rendimento definidos anteriormente.

O primeiro passo da análise discriminante consiste na confirmação de que os fatores indicados realmente diferenciam os grupos. A Tabela 8 fornece os resultados do teste de igualdade de médias entre os grupos para os diferentes setores da economia.

Tabela 8: Teste de igualdade de médias entre os grupos de rendimento

\begin{tabular}{lccc}
\hline \multicolumn{1}{c}{ Lambda de Wilks } & Primário & Secundário & Terciário \\
\hline Variáveis & Valor & Valor & Valor \\
\hline Escolaridade & $0,947^{*}$ & $0,904^{*}$ & $0,883^{*}$ \\
Experiência & $0,996^{*}$ & $0,997^{*}$ & $0,996^{*}$ \\
Masculino & $0,998^{*}$ & $0,984^{*}$ & $0,987^{*}$ \\
Branco & $0,967^{*}$ & $0,971^{*}$ & $0,964^{*}$ \\
Rural & $0,988^{*}$ & $0,993^{*}$ & $0,994^{*}$ \\
Região & $0,996^{*}$ & $0,976^{*}$ & $0,982^{*}$ \\
\hline
\end{tabular}

Fonte: Resultados da pesquisa. *Significativo ao nível de $1 \%$ pelo teste $\mathrm{F}$.

Os resultados corroboram com a análise descritiva de que as variáveis escolhidas para a análise de fato discriminam os grupos de rendimento definidos. Todas as variáveis rejeitaram a hipótese nula de igualdade de médias, é o que sugere o asterisco indicando a significância das mesmas.

O segundo passo da análise é verificar se a hipótese de igualdade entre as matrizes de variâncias e covariâncias entre grupos é confirmada. Essa hipótese é um dos pressupostos da análise discriminante linear, caso a mesma não seja confirmada outras ferramentas como a análise discriminante quadrática, logística ou do k-ésimo vizinho mais próximo devem ser utilizadas. A Tabela 9 mostra os resultados do teste $M$ de Box que possui hipótese nula de igualdade das matrizes de variâncias e covariâncias entre grupos.

Tabela 9: Teste de igualdade de matrizes de variâncias e covariâncias entre grupos

\begin{tabular}{lcc}
\hline Setor & M de Box & Nível de Significância \\
\hline Primário & 602,721 & 0,000 \\
Secundário & 3481,800 & 0,000 \\
Terciário & 10858,657 & 0,000 \\
\hline
\end{tabular}

Fonte: Resultados da pesquisa. 


\section{Revista \\ UNEMAT de \\ Contabilidade}

Pelo resultado do teste $M$ de Box evidencia-se que não é possível afirmar que o pressuposto de igualdade de variâncias e covariâncias seja válido para os grupos de rendimento no trabalho para nenhum dos setores. Portanto, o natural seria considerar a possibilidade de utilizar a análise discriminante quadrática ao invés da linear, contudo, segundo Mingoti (2005) o convencional seria estimar tanto a quadrática quanto a linear e optar pela que apresentasse maior poder de classificação. Pelo exposto optou-se pela análise discriminante linear.

O terceiro passo refere-se à estimação e validação das funções discriminantes. Com 5 grupos de rendimentos definidos é possível obter 4 funções discriminantes para cada setor de atividade. A Tabela 10 apresenta os resultados obtidos, coeficientes padronizados, autovalores e teste de significância das funções discriminantes para o setor primário.

Tabela 10: Funções discriminantes padronizadas para o setor primário, Brasil - 2011

\begin{tabular}{lrrrr}
\hline Funções & $\mathbf{1}$ & $\mathbf{2}$ & $\mathbf{3}$ & $\mathbf{4}$ \\
\hline Variáveis & Coeficientes & Coeficientes & Coeficientes & Coeficientes \\
\hline Escolaridade & 0,859 & 0,466 & 0,331 & 0,350 \\
Experiência & 0,623 & 0,574 & 0,489 & $-0,454$ \\
Masculino & 0,141 & $-0,242$ & $-0,248$ & 0,484 \\
Branco & 0,250 & 0,098 & 0,106 & 0,377 \\
Rural & $-0,189$ & $-0,384$ & 0,865 & 0,246 \\
Região & $-0,322$ & 0,858 & 0,130 & 0,538 \\
\hline Autovalores & $\mathbf{0 , 1 4 3}$ & $\mathbf{0 , 0 0 7}$ & $\mathbf{0 , 0 0 1}$ & $\mathbf{0 , 0 0 0}$ \\
\% da variância (explicada) & 94,86 & 4,46 & 0,66 & 0,02 \\
\% da variância (acumulado) & 94,86 & 99,32 & 99,98 & 100,00 \\
\hline Lambda de Wilks & 0,868 & 0,992 & 0,999 & 1,000 \\
Significância & $\mathbf{0 , 0 0 0}$ & $\mathbf{0 , 0 0 0}$ & $\mathbf{0 , 0 5 2}$ & $\mathbf{0 , 9 4 2}$ \\
\hline
\end{tabular}

Fonte: Resultados da pesquisa.

Pela análise da Tabela 10 é possível observar os coeficientes padronizados das quatro funções discriminantes assim como sua importância relativa para discriminar os grupos de rendimento (autovalores) e sua significância estatística (Lambda de Wilks). Os coeficientes formam a função que discrimina os grupos, porém eles não representam a importância de cada variável para discriminar os grupos de rendimento, que pode ser observado pelas cargas discriminantes expostas mais adiante.

Para o setor primário, a primeira função explica 94,86\% da variância entre os grupos, em outras palavras, essa é a função mais importante e a que mais discrimina os grupos de rendimentos. A segunda função explica apenas $4,46 \%$ da variância entre os grupos. As funções 1 e 2 foram significativas ao nível de $1 \%$ de acordo com o Lambda de Wilks, portanto, as funções mostram-se adequadas para classificar tanto os indivíduos da amostra como os que eventualmente sejam novos nos diferentes grupos de acordo com suas características.

A Tabela 11 apresenta os resultados obtidos, coeficientes padronizados, autovalores e teste de significância das funções discriminantes para o setor secundário. As informações apresentadas na Tabela 11 são semelhantes às da tabela anterior.

Tabela 11: Funções discriminantes padronizadas para o setor secundário, Brasil - 2011 


\begin{tabular}{lrrrr}
\hline Funções & $\mathbf{1}$ & $\mathbf{2}$ & $\mathbf{3}$ & $\mathbf{4}$ \\
\hline Variáveis & Coeficientes & Coeficientes & Coeficientes & Coeficientes \\
\hline Escolaridade & 1,088 & 0,396 & 0,206 & $-0,427$ \\
Experiência & 0,728 & 0,157 & 0,351 & 0,232 \\
Masculino & 0,431 & $-0,558$ & 0,568 & 0,207 \\
Branco & 0,223 & 0,191 & $-0,456$ & 0,776 \\
Rural & $-0,060$ & 0,112 & 0,108 & $-0,385$ \\
Região & $-0,163$ & 0,786 & 0,430 & 0,454 \\
\hline Autovalores & $\mathbf{0 , 2 4 9}$ & $\mathbf{0 , 0 1 6}$ & $\mathbf{0 , 0 0 0}$ & $\mathbf{0 , 0 0 0}$ \\
\% da variância (explicada) & 93,68 & 6,12 & 0,19 & 0,01 \\
\% da variância (acumulado) & 93,68 & 99,80 & 99,99 & 100,00 \\
\hline Lambda de Wilks & 0,788 & 0,983 & 0,999 & 1,000 \\
Significância & $\mathbf{0 , 0 0 0}$ & $\mathbf{0 , 0 0 0}$ & $\mathbf{0 , 0 2 0}$ & $\mathbf{0 , 7 5 7}$ \\
\hline Fon
\end{tabular}

Fonte: Resultados da pesquisa.

Para o setor secundário, a primeira função explica 93,68\% da variância entre os grupos. A segunda função explica apenas $6,12 \%$ da variância entre os grupos. As funções 1 e 2 foram significativas ao nível de $1 \%$ de acordo com o Lambda de Wilks, portanto, as funções mostram-se adequadas para classificar tanto os indivíduos da amostra como os que eventualmente sejam novos nos diferentes grupos.

A Tabela 12 apresenta os resultados obtidos, coeficientes padronizados, autovalores e teste de significância das funções discriminantes para o setor terciário.

Tabela 12: Funções discriminantes padronizadas para o setor terciário, Brasil - 2011

\begin{tabular}{lrrrr}
\hline Funções & $\mathbf{1}$ & $\mathbf{2}$ & $\mathbf{3}$ & $\mathbf{4}$ \\
\hline Variáveis & Coeficientes & Coeficientes & Coeficientes & Coeficientes \\
\hline Escolaridade & 1,111 & 0,266 & $-0,253$ & $-0,138$ \\
Experiência & 0,693 & 0,467 & 0,620 & 0,065 \\
Masculino & 0,306 & $-0,423$ & 0,552 & $-0,280$ \\
Branco & 0,178 & 0,031 & 0,132 & 0,713 \\
Rural & $-0,042$ & $-0,020$ & $-0,033$ & 0,639 \\
Região & $-0,136$ & 0,831 & 0,090 & $-0,006$ \\
\hline Autovalores & $\mathbf{0 , 3 9 0}$ & $\mathbf{0 , 0 0 4}$ & $\mathbf{0 , 0 0 1}$ & $\mathbf{0 , 0 0 0}$ \\
\% da variância (explicada) & 98,69 & 1,08 & 0,23 & 0,00 \\
\% da variância (acumulado) & 98,69 & 99,77 & 100,00 & 100,00 \\
\hline Lambda de Wilks & 0,716 & 0,995 & 0,999 & 1,000 \\
Significância & $\mathbf{0 , 0 0 0}$ & $\mathbf{0 , 0 0 0}$ & $\mathbf{0 , 0 0 0}$ & $\mathbf{0 , 6 8 4}$ \\
\hline
\end{tabular}

Fonte: Resultados da pesquisa.

Para o setor terciário, a primeira função explica 98,69\% da variância entre os grupos. As funções 2 e 3 explicam apenas $1,08 \%$ e $0,23 \%$ da variância entre os grupos, respectivamente. As funções 1, 2 e 3 foram significativas ao nível de $1 \%$ de acordo com o Lambda de Wilks. Novamente, as funções são adequadas para classificar tanto os indivíduos da amostra como os que eventualmente sejam novos nos diferentes grupos.

Em resumo, as funções discriminantes mais importantes para diferenciar os grupos de rendimento de cada setor podem ser definidas como: 


\section{Contabilidade}

$\left\{\begin{array}{c}\text { Primário: } \mathrm{Y}=0,859 \mathrm{Esc}+0,623 \mathrm{Exp}+0,141 \mathrm{Masc}+0,250 \mathrm{Bra}-0,189 \mathrm{Rur}-0,322 \mathrm{Reg} \\ \text { Secundário: } \mathrm{Y}=1,088 \mathrm{Esc}+0,728 \mathrm{Exp}+0,431 \text { Masc }+0,223 \mathrm{Bra}-0,060 \mathrm{Rur}-0,163 \mathrm{Reg} \\ \text { Terciário: } \mathrm{Y}=1,111 \mathrm{Esc}+0,693 \mathrm{Exp}+0,306 \text { Masc }+0,178 \mathrm{Bra}-0,042 \mathrm{Rur}-0,136 \mathrm{Reg}\end{array}\right.$

Por fim, o quarto passo consiste na análise do poder de classificação do modelo quanto aos indivíduos e grupos e também na definição de quais variáveis são mais importantes para a discriminação entre grupos. A Tabela 13 apresenta os centroides dos grupos de acordo com a primeira função discriminante de cada setor, devido a mesma ter apresentado maior importância relativa.

Tabela 13: Centroides dos grupos de rendimento de acordo com a primeira função

\begin{tabular}{lrrr}
\hline Centroides dos grupos & Primário & Secundário & Terciário \\
\hline até 2 salários mínimos & -0.167 & -0.347 & -0.492 \\
de 2 a 4 salários mínimos & 0.678 & 0.392 & 0.322 \\
de 4 a 10 salários mínimos & 0.967 & 0.939 & 0.941 \\
de 10 a 20 salários mínimos & 1.176 & 1.513 & 1.416 \\
mais de 20 salários mínimos & 1.178 & 1.214 & 1.266 \\
\hline
\end{tabular}

Fonte: Resultados da pesquisa.

O centroide de cada grupo de rendimento pode ser interpretado como uma medida de distância. Nesse caso, fatores com relação positiva aumentam a chance do indivíduo estar classificado nas faixas mais altas de renda conforme a variável aumenta. Quanto aos fatores com relação negativa, esses aumentam a chance do indivíduo estar nas classes mais baixas de renda de acordo com o aumento da variável.

A Tabela 14 expõe as cargas discriminantes de cada fator de acordo com a primeira função discriminante de cada setor, devido a mesma ter apresentado maior importância relativa. As cargas discriminantes são a correlação entre a variável e a função discriminante, portanto, quanto maior a correlação mais importante é a variável para a função.

Tabela 14: Cargas discriminantes de acordo com a primeira função

\begin{tabular}{lrrr}
\hline Cargas Discriminantes & Primário & Secundário & Terciário \\
\hline Escolaridade & 0,626 & 0,648 & 0,716 \\
Experiência & 0,144 & 0,107 & 0,089 \\
Masculino & 0,092 & 0,204 & 0,180 \\
Branco & 0,484 & 0,342 & 0,310 \\
Rural & $-0,266$ & $-0,165$ & $-0,123$ \\
Região & $-0,585$ & $-0,263$ & $-0,203$ \\
\hline Taxa de acerto $(\%)$ & $\mathbf{8 3 , 0 0}$ & $\mathbf{6 4 , 7 0}$ & $\mathbf{6 1 , 0 0}$ \\
\hline
\end{tabular}

Fonte: Resultados da pesquisa.

Como esperado a variável mais importante para discriminar o rendimento pessoal nos três setores foi a escolaridade. No setor terciário a escolaridade chega a ter o dobro de importância que a segunda variável mais importante. Portanto, assim como Hanushek et al (2001) é possível afirmar que a educação é um significativo instrumento de transferência e redistribuição de renda, em outras palavras, investimentos em educação tem efetividade no combate à desigualdade e a pobreza. 


\section{Revista \\ UNEMAT de \\ Contabilidade}

v. 9, n. 17,2020

Como observado anteriormente o setor terciário, o menos desigual, apresenta maior nível médio de escolaridade, evidenciando, dessa forma, que a educação de fato pode contribuir para a melhoria na distribuição de renda. Contudo, como se pode observar da Tabela 14, outros fatores também exercem influência significativa para a discriminação entre os grupos.

No setor primário, por exemplo, as variáveis região e branco são a segunda e terceira mais importantes para explicar a diferença entre grupos. Trabalhar na Região Nordeste (região igual a 5) aumenta as chances de encontrar-se nas classes mais baixas de renda enquanto ser branco tem o efeito contrário. Já nos setores secundário e terciário, depois de escolaridade, ser não branco e trabalhar na Região Nordeste também afetam de forma importante a chance de estar nas faixas mais baixas de renda.

A Tabela 14 ainda contém informação sobre a taxa de acerto do modelo em cada setor. Dada a discrepância de observações entre os grupos, as probabilidades de cada grupo foram ajustadas de acordo com o tamanho. O setor primário obteve taxa de acerto de $83 \%$, a mais elevada, o setor secundário com $64,70 \%$ e o terciário com $61 \%$ obtiveram taxa de acerto menor. Cabe ressaltar que dado o grande número de observações, a discrepância entre os grupos em termos de tamanho e o fato de variáveis importantes para a definição de salários como o treinamento no trabalho não estarem presentes, consideram-se razoáveis as taxas de acerto dos modelos.

\section{CONSIDERAÇÕES FINAIS}

A desigualdade na distribuição de renda é sempre apontada como um dos principais responsáveis pelos contrastes sociais observados no Brasil. O país é classificado como em desenvolvimento de renda média alta e está entre as dez maiores economias do mundo. No entanto, apresenta indicadores de pobreza e de distância entre ricos e pobres incompatíveis com essa posição.

Além da desigualdade na distribuição de renda, são notáveis o gap educacional e as discrepantes oportunidades no mercado de trabalho envolvendo pessoas de raças e gêneros diferentes. Contudo, estudos têm sugerido que investimentos em educação podem ser ferramentas de transferência e redistribuição de renda eficazes.

Os resultados do presente artigo permitiram identificar a existência de desigualdade na distribuição de renda nos setores primário, secundário e terciário. A desigualdade foi menor no setor que possuía o maior nível de escolaridade média. Além disso, evidenciou-se que a escolaridade é o principal fator que explica as diferenças de renda entre os grupos de rendimentos formados.

Nesse caso, a educação realmente é o principal instrumento que pode reduzir a desigualdade no Brasil. Dessa forma, é necessário investimento em educação para elevar o estoque de capital humano do país. Ademais, variáveis como gênero, raça e local de residência também são relevantes para discriminação da renda, entretanto, como estão associados a fatores culturais são de difícil modificação. Esse fato está associado a discriminação no mercado de trabalho que também deve ser levada em consideração para mudanças nas estruturas de distribuição de renda no Brasil.

\section{REFERÊNCIAS}


ADAMS, Jr. R. H. Economic growth, inequality and poverty: Estimating the growth elasticity of poverty. World Development. v. 32, n.12, p. 1989-2014. 2004.

ALTONJI, J. G.; BLOM, E.; MEGHIR, C. Heterogeneity in human capital investments: High school curriculum, college major, and careers. NBER Working Paper n. 17985. 2012.

BECKER, G. S. Investment in human capital: A theoretical Analisys. Journal of Political Economy. v. 70, n. 5, p. 9-49, 1962.

CARVALHO, A. P. de.; NERI, M. C.; SILVA, D. B. Diferenciais de Salários por Raça e Gênero: Aplicação dos procedimentos de Oaxaca e Heckman em Pesquisas Amostrais Complexas. XV Encontro de Estudos Populacionais, 18 a 22 setembro de 2006, ABEP. 2006.

COURSEIL, C. H.; SANTOS, D. D.; FOGUEL, M. N. Decisões críticas em idades críticas: A escolha dos jovens entre estudo e trabalho no Brasil e em outros países da América Latina. Texto para Discussão n. 797. 2001.

GLAESER. E. L.; HANUSHEK, E. A.; QUIGLEY, J. M. Opportunities, race, and urban location: The influence of John Kain. NBER Working Paper n. 10312. 2004.

HAIR, Jr. J. F.; ANDERSON, R. E.; TATHAM, R. L.; BLACK, W. C. Análise multivariada de dados. Bookman - Porto Alegre, 2005.

HANUSHEK, E. A.; LEUNG, C. K. Y.; YILMAZ, K. Redistribution through education and others transfer mechanisms. NBER Working Paper n. 8588. 2001.

HANUSHEK, E. A.; WOESSMANN, L. Schooling, cognitive skills, and the Latin American growth puzzle. NBER Working Paper n. 15066. 2009.

MINCER, J. Schooling, experience, and earnings. National Bureau of Economic Research: Columbia University Press, New York, 1974.

MINGOTI, S. A. Análise de dados através de métodos de estatística multivariada: Uma abordagem aplicada. Editora UFMG - Belo Horizonte, 2005.

NERI, M.; CARVALHAES, L, Será esta década, a da igualdade, Conjuntura Econômica, v, 62, n, 10, p, 48-51, 2008

OAXACA, R. Male-Female Wage Differentials in Urban Labor Market. International Economic Review. v. 14, n. 3, p. 693-709. 1973.

SILVEIRA, G. F. Retornos da escolaridade no Brasil e Regiões. Universidade Federal de Viçosa, Dissertação. Viçosa, 2011.

SOUZA, A. P.; PONCZEK, V.; OLIVA, B. Os determinantes do fluxo escolar entre o ensino fundamental e o ensino médio no Brasil. Texto para Discussão 286 - FGV. 2011.

TODARO, M. P.; SMITH, S. S. Economic development, 11 $^{\text {th }}$ ed. Prentice Hall, 2012. 